\title{
Comparison of different copula assumptions and their application in portfolio construction
}

\author{
František ŠTULAJTER, ČSOB AM, Slovak Republic ${ }^{i}$
}

\begin{abstract}
The paper deals with modeling of mutual dependencies among financial assets. Its aim is to investigate the impact of different copula assumptions on optimal portfolios, when CVaR optimization is used. Strategic asset allocation perspective is supposed. It is demonstrated that copula functions enable us to separate the modeling of dependency features of financial assets from the modeling of marginal distribution characteristics, in the context of practical portfolio construction tasks. The difference between portfolios constructed using normal copula and student $t$ copula is shown when mutual or pension fund exposed to long-only constrain is assumed. The fund is considered to invest solely into equity and fixed-income instruments. As expected, the exclusive use of linear correlation coefficients leads to underestimation of total portfolio risk. The superiority of student $t$ copula portfolios intensifies as the confidence level of CVaR rises and/or as the CVaR target increases.
\end{abstract}

\section{Keywords}

Copula functions, correlation, CVaR, financial modeling, portfolio construction

JEL Classification: C10, G11

\footnotetext{
${ }^{\mathrm{i}}$ CSOB Asset Management, Kolarska 6, 81563 Bratislava, Slovak Republic. fstulajter@csob.sk
}

\section{Introduction}

Financial applications often deal with the multivariate distributions of random numbers. The obvious task for financial modelers is to describe the features and behavior of these multivariate distributions. Statistical properties of financial data are a central point of portfolio construction and evaluation problems, which constitute the generic asset allocation problem.

Following Meucci (2005), the quantitative framework of solving a generic asset allocation problem can be summarized to several steps: (i) detecting quantities that fully describe behavior of asset prices: so-called market invariants. For equities the invariants are the returns; for bonds the invariants are the changes in the yield to maturity, for vanilla derivatives it is the change in implied volatility; (ii) estimating the distribution of market invariants; (iii) mapping the distribution of market invariants into the distribution of asset prices at a generic time in the future; (iv) defining optimality depending on investor's profile; ( $v$ ) computing the optimal allocation, solving portfolio selection problem. Further, the risk estimation can be incorporated. Ability to correctly describe and estimate properties of financial asset returns are crucially important to successfully solve asset allocation problems. If the distribution of market invariants is assumed to be multivariate normal, the estimation process and optimal portfolio optimization is straightforward. However, distributions of market invariants are usually fat-tailed and skewed. Not only do marginal characteristics not conform to normality assumption, but the dependencies are source of confusion as well.

There can be found many drawbacks of "normal" specification of the dependency structure in the nonGaussian world. Embrechts et al. (2002) identified and illustrated several major problems associated with a correlation coefficient, defined as Pearson's product moment: 
- The correlation coefficient is a measure of linear association of random variables and as such, it cannot capture non-linear dependencies.

- Feasible values for the correlation depend on the marginal distributions. Due to the specification of correlation as a scaled covariance, the correlation coefficient will always be influenced by the distribution of marginals.

- Perfect positive dependence does not imply a correlation of one.

- Zero correlation does not imply independence.

Another problem is that the correlation describes the dependency as one single number rather than expressing it as some functional (e.g. the returns of financial assets usually posses higher dependency in lower tails of their multivariate distribution).

There are two major methods how to work with non-normal distributions in finance, namely static and dynamic method. Static method assumes that there exists a generic multivariate distribution of market invariants, which is unobservable. The dynamic method in the simplest form works with conditional normality assumption and tries to describe dynamically changing parameters of this distribution. This article focuses on static method and copula functions as tool to describe the dependencies among market invariants and theoretical concept to overcome correlation coefficients shortcomings.

Pitfalls of the correlation coefficient lead to many failures of financial applications. In risk management or actuarial finance, the estimates of risk metrics, like Value at Risk, possibly underestimate risks. In the area of portfolio management, the optimal portfolios could be far less diversified than expected. As a result, a new assessment of dependency modeling for finance was proposed in the last decade of the $20^{\text {th }}$ century.

Sklar's theorem is used to show the existence of a specific function called copula, which links together pure univariate features of marginal distributions into the whole multivariate distribution. The copula function enables to independently model the specification of marginal distributions and the purely joint features. In this context, stronger tail dependency can be easily justified as well as skewed behavior of random numbers.

A comprehensive introduction to copula functions can be found in Embrechts (2008) as well as a list of copula must-reads. Copula methods in finance attracted a lot of attention in recent years. Thorough analysis of this subject can be found in the publications of Alexander (2008) or Nelsen (2006). Financial applications of copula methods are covered in papers by Bouyé et al. (2000), Chorós et al. (2009) or the publication of Cherubini et al. (2004). Interesting application of copula methods in hydrology is mentioned by Genest and Favre (2007).

As copula tries to overcome pitfalls of correlation coefficients, new risk measures were developed to assign more emphasis on extreme risks. Downside risks measures like Value at Risk or its extension Expected Shortfall are the most popular ones. They focus on relaxing strong assumptions of Modern Portfolio Theory and the framework of mean-variance analysis and mean-variance optimization.

The foundations of portfolio selection problem were made by Markowitz in his paper Portfolio Selection published in 1952. As is shown in Fabozzi et al. (2006) mean-variance approach is consistent with: (1) expected utility maximization under quadratic utility assumptions, or (2) the assumption that security returns are jointly normally distributed.

Blindly following assumption of joint normality has caused failures in risk assessment in many financial institutions; see e.g. Stulz (2008). The development of new risk measure was most extensive at JP Morgan. The Risk metrics technical documents and introduced Value at Risk framework became standard in the field. Despite its wide use, VaR has several undesirable properties, well documented in literature; see e.g. Rau-Bredow (2004). The set of natural properties that a reasonable measure of risk should satisfy was proposed by Artzner et al. (1999).

This led to the introduction of a set of risk measures that are based on estimating the mean value of losses, which exceed VaR value. These measures are referred to as (i) Mean Excess Loss or Expected Shortfall when expected losses strictly exceed VaR; (ii) Tail VaR when expected losses weakly exceed VaR and finally (iii) Conditional Value at Risk $(\mathrm{CVaR})$ given as weighted average of $\mathrm{VaR}$ and Expected Shortfall; see e.g. Rockafellar and Uryasev (2000). Optimization framework utilizing CVaR was proposed by Rockafellar and Uryasev (2000). An attractive formulation of their solution can also be found in Meucci (2006).

Value at Risk method armed with copula dependency structure description is subject to many studies. Palaro and Hotta (2006) proposed using copula functions to model GARCH innovations when $\mathrm{VaR}$ is being estimated. The authors show the superiority of the Joe-Clayton copula. Bai and Sun (2007) describe the copula theory in relation to $\mathrm{CVaR}$ measures. The GARCH model with three-dimensional Archimedean copula is investigated. Integrated risk management with emphasis on credit risk is scrutinized in He and Gong (2009). The copula based $\mathrm{CVaR}$ model for market and credit risk is used. 
The objective of this paper is to point out the differences between two copula function assumptions in portfolio construction context. We considered two dependency structure assumptions; normal copula and student $t$ copula. The real world examples are used to emphasize the need of a copula modeling framework when estimating optimal portfolios with Conditional Value at Risk chosen as the risk measure. This problem is solved from a strategic asset allocation perspective. Portfolios are exposed to long-only investment constraints and invest solely into equity and fixed-income instruments. Monte Carlo simulations are utilized to estimate optimal portfolios. Impact of different confidence levels and different CVaR targets are investigated to emphasize the differences between optimal portfolios. The CVaR approach is chosen as a tool to estimate optimal portfolios and complement analysis of different dependency structure assumptions.

The paper is organized as follows. The next section defines copula functions together with the most important topics: its representation, elliptical and Archimedean copulas and estimation. Firstly, the need of advanced dependency structure models is supported by empirical observations. In the third section, the Conditional VaR optimization problem is briefly introduced. In the fourth part we show numerical examples which demonstrate the importance of copula modeling in portfolio construction. The pure linear correlation model (or normal copula model) is confronted with student $\mathrm{t}$ copula assumption. Differences among optimal portfolios estimated under these assumptions are shown in various characterizations of optimization problem. The last part concludes.

In the article the terms copula and copula function will be used interchangeably. We try to offer a valuable introduction to copula modeling framework in the context of portfolio management and always attempt to provide the interested reader with standard references.

\section{Dependency patterns of financial data - need of copulas}

The fat-tailed character of marginal distributions of market invariants is well known and observed from most of the empirical data. The asymmetric dependencies within implied volatilities are obvious as well. However the asymmetry of market invariants of equities and bonds, namely of returns and changes in yields to maturity, can be questioned. To graphically depict the pure dependency structure between different time series of market invariants the gradegrade chart is created. It is the scatter plot of percentiles, which are called grades.
Empirical cumulative distribution functions are used to estimate percentiles. In this way, marginal characteristics of data are totally filtered out. Every point in the chart shows the combination of percentiles of two invariants. Combinations of low percentiles represent the market observation of parallel negative realization of market invariants. In other words, both time series realize returns (in the case of equities) from the left tail of their marginal distributions.

Figure 1 represents the dependency structure of weekly returns of stock indexes S\&P 500 and DJ Stoxx 600 dated from $30^{\text {th }}$ December 1988 to $17^{\text {th }}$ July 2009. The total number of observations is thus 1073 . The sample correlation coefficient of these indexes when estimated using over 20 years of weekly data is 0.747 .

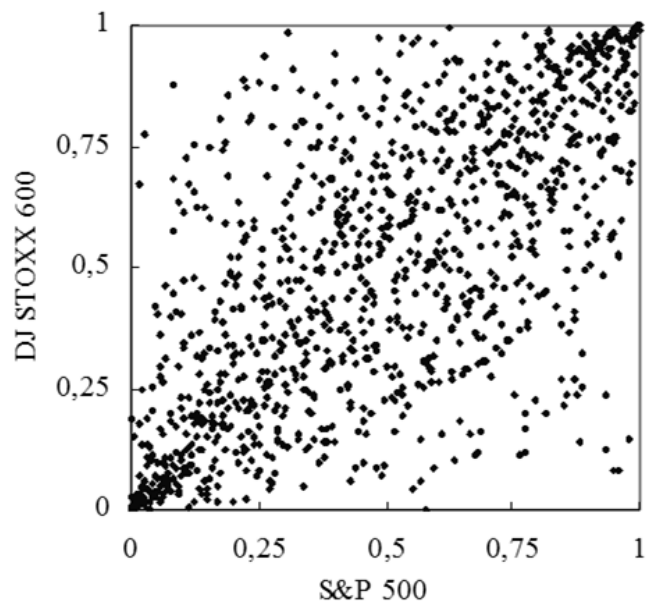

Figure 1 Grade-grade chart of S\&P 500 and DJ Stoxx 600

Figure 2 shows the scatter plot of two normally distributed random numbers, $a$ and $b$, with the same correlation and the same number of observations. The stronger tail dependence (occurrence of returns in the tails of marginal distribution) of real world returns is obvious.

The observations in Figure 1 are more concentrated to the downward left corner of the chart which represents stronger tail dependency than would have been predicted by multivariate normal distribution with sample correlation coefficient (by normal copula as will be seen in the following sections).

To more precisely define the shown differences, the total number of observations in the various downward left corners was calculated as a fraction of all data points. This quantity is related to quantiledependent measure of dependence introduced by Coles et al. (1999) which exactly defines the probability that one variable is extreme given that the other is also extreme. It is also known as lower tail 


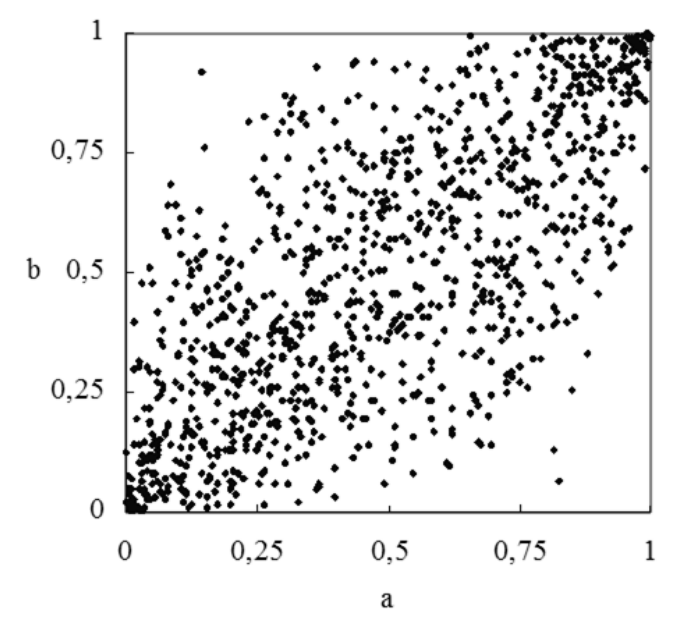

Figure 2 Grade-grade chart of normally distributed random numbers with $\rho=0.747$

dependence coefficient. Three different "corners" were assumed; namely $20^{\text {th }}-20^{\text {th }}$ percentile, $10^{\text {th }}-10^{\text {th }}$ percentile and $5^{\text {th }}-5^{\text {th }}$ percentile corner. These quantities represent the total number of joint observations of both variables that are lesser than $\mathrm{x}^{\text {th }}$ percentiles. In the case of real financial data the percentiles are given as empirical estimates. For normally distributed random numbers, Monte Carlo simulation was used to calculate the number of observations in the downward left corners assuming empirical correlations. Normal cumulative density function was used to calculate "normal" percentiles. As marginal characteristics of empirical and random numbers are filtered out, only pure dependency structure is investigated. Table 1 shows the defined quantities for different equity and fixed income invariants:

The hypothesis of stronger tail dependency cannot be rejected as the ratio of empirical tail frequency and normal copula frequency occurrences is bigger than one except in the case of the American and Japanese equity index. The grade-grade charts of invariant pairs from Table 1 can be found in Appendix B as well as grade-grade charts of selected dependency structures when daily data sets are used. There are presented charts representing dependency structures of different equity markets as well as changes of generic 2 and 10 year US treasury bonds' yields. The same amount of financial data is used as in the previous example. Appendix A shows relative frequencies of different dependency structure assumptions when daily data sets are used. Appendix C contains a description of data used to calculate quantities from Table 1 as well as a description of data used in examples in Section 4.

\subsection{Definition of copulas}

The distribution of a multivariate random variable $X$ can be factored into two separate components. The marginal distributions of each entry vector $X$ represent the purely univariate features of $X$. On the other hand, the purely joint component of the distribution of $X$ can be summarized in standardized distribution, copula. The copula represents the true interdependence structure of the random variable. Intuitively, the copula is a standardized version of the purely joint features of the multivariate distribution, which is obtained by filtering out all the purely onedimensional features, namely the marginal distributions of each entry $X_{n}$.

In order to factor out the marginal components, it is necessary to deterministically transform each entry $X_{n}$ in a new random variable $U_{n}$, whose distribution is the same for each entry. Since all $U_{n}$ have the same distribution, the univariate features of $X$ are removed. It is natural in financial modeling to consider cumulative distribution function $F_{X}$ to map a generic random variable $X$ into a random variable $U$. Following Meucci (2005) the random variable $U$ is called grade of $X$ and reads:

$$
U=F_{X}(X) .
$$

The grade of $X$ is a deterministic transformation of the random variable $X$ that assumes values in the interval $[0,1]$. In particular, each marginal component $X_{n}$ can be standardized by means of uniform distribution. The random variable $U$ can be expressed as the vector of the grades:

Table 1 Relative frequencies of different dependency structure assumptions

\begin{tabular}{|l|c|c|c|c|c|c|c|}
\hline & & \multicolumn{3}{|c|}{ Empirical tail frequency } & \multicolumn{3}{|c|}{ Normal copula tail frequency } \\
\hline Invariants pair & $\rho$ & 5 th -5 th & 10 th -10 th & 20 th -20 th & 5 th -5 th & 10 th -10 th & 20 th -20 th \\
\hline S\&P 500 - DJ STOXX 600 & 0.747 & $2.89 \%$ & $5.88 \%$ & $12.69 \%$ & $2.16 \%$ & $4.93 \%$ & $11.80 \%$ \\
\hline S\&P 500 - NASDAQ 100 & 0.834 & $3.08 \%$ & $6.44 \%$ & $14.55 \%$ & $2.70 \%$ & $5.77 \%$ & $13.51 \%$ \\
\hline S\&P 500 - NIKKEI 225 & 0.455 & $1.12 \%$ & $3.36 \%$ & $8.86 \%$ & $1.16 \%$ & $2.91 \%$ & $8.01 \%$ \\
\hline DJ STOXX 600 - NIKKEI 225 & 0.521 & $1.49 \%$ & $4.29 \%$ & $9.89 \%$ & $1.24 \%$ & $3.25 \%$ & $8.79 \%$ \\
\hline US 2YR TR - US 10YR TR & 0.822 & $2.80 \%$ & $6.25 \%$ & $13.99 \%$ & $2.64 \%$ & $5.59 \%$ & $13.26 \%$ \\
\hline
\end{tabular}




$$
U=\left(\begin{array}{c}
U_{1} \\
\vdots \\
U_{N}
\end{array}\right)=\left(\begin{array}{c}
F_{X_{1}}\left(X_{1}\right) \\
\vdots \\
F_{X_{N}}\left(X_{N}\right)
\end{array}\right)
$$

In other words, the random variable $U$ represents percentiles of random variable $X$. The copula of the multivariate random variable $X$ is the joint distribution of its grades. Sklar's theorem shows, that given any multivariate random variable $X$ with continuous marginal distributions, there is a unique copula function $\mathrm{C}$ such that:

$$
F_{X}\left(x_{1}, \ldots, x_{N}\right)=C\left(F_{X_{1}}\left(X_{1}\right), \ldots F_{X_{N}}\left(X_{N}\right)\right) .
$$

Corresponding probability density function of the multivariate random variable can be expressed as the product of the pdf of copula and the pdf of the marginal densities of its entries:

$$
f_{x}\left(x_{1}, \ldots, x_{N}\right)=f_{C}\left(F_{X_{1}}\left(x_{1}\right), \ldots F_{X_{N}}\left(x_{N}\right)\right) \prod_{n=1}^{N} f_{X_{n}}\left(x_{n}\right) \text {. }
$$

Since the copula is a distribution, namely distribution of the grades $U$ (or percentiles of the random number realization) it can be represented in terms of the probability density function or the cumulative distribution function, or the characteristic function. It is proved in appendix www.2.3 of Meucci (2005) that the pdf of the copula reads:

$$
f_{C}\left(u_{1}, \ldots, u_{N}\right)=\frac{f_{X}\left(Q_{X_{1}}\left(u_{1}\right), \ldots, Q_{X_{N}}\left(u_{N}\right)\right)}{f_{X_{1}}\left(Q_{X_{1}}\left(u_{1}\right)\right) \ldots . . f_{X_{N}}\left(Q_{X_{N}}\left(u_{N}\right)\right)},
$$

where $Q_{X_{n}}$ is the quantile function (equivalent to inverse cdf) of generic $n$-th marginal entry of $X$. And the cdf of the copula reads:

$$
F_{C}\left(u_{1}, \ldots, u_{N}\right)=F_{X}\left(Q_{X_{1}}\left(u_{1}\right), \ldots, Q_{X_{N}}\left(u_{N}\right)\right) .
$$

The copula of random variable $X$ could be equivalently represented in terms of its characteristic function as well.

There are two more concepts, central to the copula functions theory; tail dependence and bounds for dependence. The lower/upper tail dependence coefficient represents the conditional probability that one random variable takes a value in its lower/upper tail, given that the other random variable takes a value in its lower/upper tail. Copula is said to have symmetric tail dependence if their lower and upper tail dependence coefficients are equal. Conversely, it has asymmetric tail dependence if its tail dependence coefficients differ. There exist independence copulas, copulas with perfect positive dependence and copulas with perfect negative dependence. Perfect positive/negative dependency is defined as Fréchet upper/lower bound copulas. No other copula can take a value that is greater than the value of Fréchet upper bound copula and no other copula can take a value that is less than the value of Fréchet lower bound copula. As it is highlighted in Alexander (2008), less than perfect (positive or negative) dependence is linked to certain parametric copulas. Copula captures positive or negative dependence between the variables if it tends to one of Fréchet bounds as its parameter values change. The Gaussian copula does not tend to Fréchet upper bound as the correlation increases to 1 and neither does it tend to Fréchet lower bound as approaching correlation of -1 .

\subsection{Copula families}

There exist many methods to derive copula functions; see e.g. Nelsen (2006). The most commonly used are the inversion method and generator functions method. The inversion method derives copula representations from multivariate distributions such as normal or student $\mathrm{t}$ distribution. The most common examples of inversion method derived copulas are elliptical copulas. An alternative method for building copulas is based on a generator function. These copulas are called Archimedean copulas.

\section{Elliptical copulas}

A normal copula is derived using the inversion method from the multivariate and univariate standard normal distribution functions, denoted $\Phi$ and $\phi$. It is defined as:

$$
C\left(u_{1}, \ldots, u_{2} ; \Sigma\right)=\Phi\left(\phi^{-1}\left(u_{1}\right), \ldots \phi^{-1}\left(u_{N}\right)\right) .
$$

The cumulative distribution function of normal copula cannot be written in a simple closed form. The normal copula probability density function is given by:

$$
c\left(u_{1}, \ldots, u_{n} ; \Sigma\right)=|\Sigma|^{-1 / 2} \exp \left(-1 / 2 \xi^{\prime}\left(\Sigma^{-1}-\mathrm{I}\right) \xi\right),
$$

where $\Sigma$ denotes the correlation matrix, $|\Sigma|$, is its determinant and $\xi=\left(\xi_{1}, \ldots, \xi_{n}\right)^{\prime}$, where $\xi_{i}$ is the $u_{i}$ quantile of the standard normal random variable $X_{i}{ }^{1}$ The only one unknown parameter is, in this case, the correlation matrix $\Sigma$. The normal copula density is calculated as follows:

- Firstly, the grades of marginals are quantified using cumulative distribution functions of corresponding marginals: $u_{i}=F_{X_{i}}\left(x_{i}\right)$ for $i=$ $1 \ldots n$. It is important to highlight that copula modeling enables to use different probability specification for every marginal. Also empiric cdf could be used.

$$
{ }^{1} u_{i}=P\left(X_{i}<\xi_{i}\right), X_{i} \sim N(0,1), i=1, \ldots, n .
$$


- Apply the quantile standardized normal function on grades: $\xi_{i}=\phi^{-1}\left(u_{i}\right)$ for $i=1 \ldots n$.

- Use the correlation matrix $\Sigma$ to calculate the normal copula density.

This sequential, two-stage copula modeling approach can be used to plausibly estimate copula functions parameters as well as to create Monte Carlo simulations where different dependency structure assumptions are utilized. Further presentation of estimation and simulation topics is covered in following sections.

Figure 3 shows the probability density function of a bivariate normal copula with correlation coefficient 0.5 as a function of $u_{1}$ and $u_{2}$ which each range from 0 to 1 . The normal copulas have symmetric tail dependence behavior, which is obvious from the graphical representation. They have zero or very weak tail dependence unless the correlation is 1 . When the marginals are also normally distributed, there is always zero tail dependency. Obviously, this is not appropriate for modeling dependencies among financial assets.

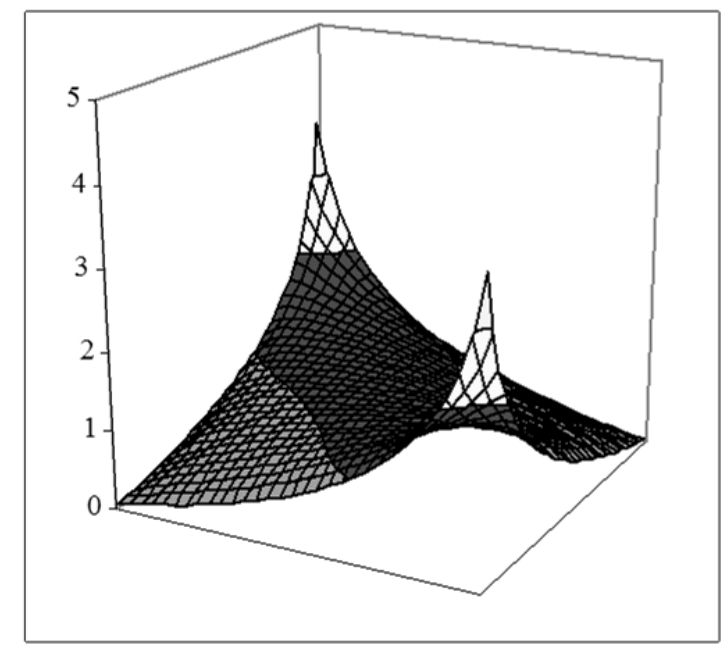

Figure 3 PDF of normal copula with $\rho=0.5$.

Source: Figure II. 6.1 reproduced from Market Risk Analysis: Practical financial econometrics, 2008, C. Alexander published by John Wiley \& Sons Ltd, with permission from the author.

Another elliptical copula, which is derived implicitly from a multivariate distribution function, is student $\mathrm{t}$ copula. It is defined as:

$$
C_{v}\left(u_{1}, \ldots, u_{n} ; \Sigma\right)=t_{v}\left(t_{v}^{-1}\left(u_{1}\right), \ldots, t_{v}^{-1}\left(u_{n}\right)\right),
$$

$t_{v}$ and $t_{v}$ are multivariate and univariate Student $\mathrm{t}$ distribution functions with $v$ degrees of freedom and $\Sigma$ denotes the correlation matrix. Like the normal copula, the student $t$ copula cumulative distribution cannot be written in a single closed form. The student $t$ copula probability density function is defined as:

$$
\begin{gathered}
c_{v}\left(u_{1}, \ldots, u_{n} ; \Sigma\right)=K|\Sigma|^{-1 / 2}\left(1+v^{-1} \xi^{\prime} \Sigma \xi\right)^{-(v+n) / 2} . \\
\prod_{i=1}^{n}\left(1+v^{-1} \xi_{i}^{2}\right)^{(v+1) / 2},
\end{gathered}
$$

where $\xi^{\prime}=\left(t_{v}^{-1}\left(u_{1}\right), \ldots, t_{v}^{-1}\left(u_{n}\right)\right) \quad$ is a vector of realizations of student t variables and $K()$ is a gamma function defined as:

$$
K=\Gamma\left(\frac{v}{2}\right)^{n-1} \Gamma\left(\frac{v+1}{2}\right)^{-n} \Gamma\left(\frac{v+n}{2}\right) .
$$

Figure 4 shows a bivariate $\mathrm{t}$ copula probability density function with 4 degrees of freedom and correlation of 0.5 also as a function of $u_{1}$ and $u_{2}$. Note that the peaks in the tails are symmetric because the copula has symmetric tail dependency but they are higher than those of normal copula with correlation of 0.5 because the $t$ copula has stronger tail dependence.

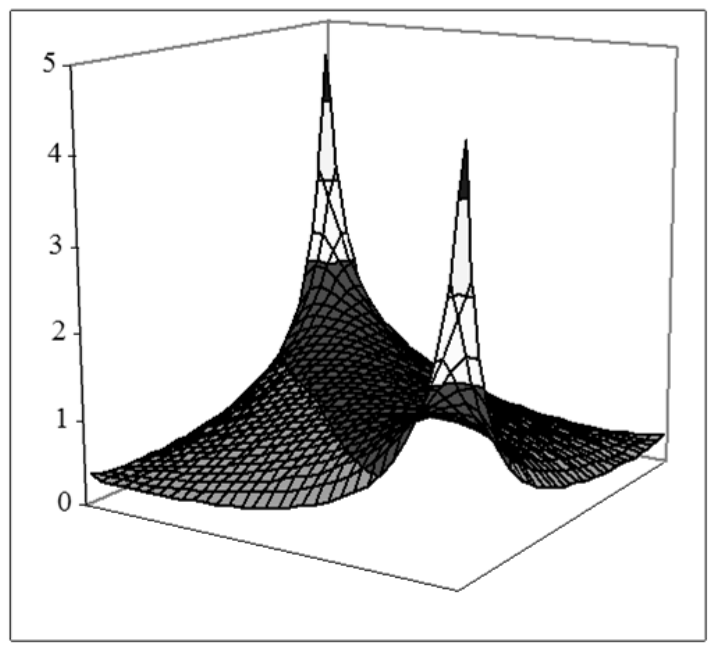

Figure $4 \mathrm{PDF}$ of Student $\mathrm{t}$ copula with $\rho=0.5$ and 4 degrees of freedom

Source: Figure II. 6.1 reproduced from Market Risk Analysis: Practical financial econometrics, 2008, C. Alexander published by John Wiley \& Sons Ltd., with permission from the author.

However, the combination of the normal copula and student $t$ marginals can "create" multivariate distribution with stronger tail dependence. Although both normal and student $t$ copulas are elliptical copulas with symmetric distributions, ${ }^{2}$ for practical purposes, a shape of the whole distribution of random variable $X$ (the joint distribution of marginals and copula) is important. The combinations of elliptical copulas and asymmetrically distributed marginals give

\footnotetext{
${ }^{2}$ There exists a wide variety of Student $t$ copulas; many of them with asymmetric tail dependence. See e.g. Demarta and McNeil (2005).
} 
rise to non-symmetrical behavior of random variables, which are particularly important in financial modeling, especially when derivatives are considered.

\section{Archimedean copulas}

Archimedean copulas are constructed using the generator functions method. Given any generator function $\Psi$, it is possible to define the corresponding Archimedean copula as:

$$
C_{A}\left(u_{1}, \ldots, u_{n}\right)=\Psi^{-1}\left(\psi\left(u_{1}\right)+\ldots+\psi\left(u_{n}\right)\right) .
$$

Its associated density function is

$$
c_{A}\left(u_{1}, \ldots, u_{n}\right)=\Psi_{(n)}^{-1}\left(\psi\left(u_{1}\right)+\ldots+\psi\left(u_{n}\right)\right) \prod_{i=1}^{n} \Psi^{\prime}\left(u_{i}\right) \text {, }
$$

where $\Psi_{(n)}^{-1}$ is the $n-t h$ derivative of the inverse generator function. When the generator function $\Psi(u)=-\ln (u)$ the Archimedean copula becomes the independent copula. More generally, the generator function can be any strictly convex and monotonic decreasing function. Hence, there exists a large number of different Archimedean copulas. Alexander (2008) introduced two simple Archimedean copulas that are used in market risk analysis thanks to its asymmetric tail dependence. These are Clayton and Gumbel copulas. The corresponding generator function of Clayton copula is defined as

$$
\Psi^{\text {Clayton }}(u)=\alpha^{-1}\left(u^{-\alpha}-1\right), \alpha \neq 0,
$$

and the generator function of Gumbel copula as

$$
\Psi^{\text {Gumbel }}(u)=-(\ln (u))^{\delta}, \delta \geq 1 .
$$

Interested readers are referred to Alexander (2008) or Nelsen (2006) for further discussion of Archimedean copulas.

\subsection{Estimation of copulas}

The multivariate distribution of the random number $X$ comprises from the univariate distributions of its marginals and the distribution of the copula. In general, it is possible to estimate the copula and marginals parameters together in one step using the so-called full Maximum Likelihood Estimation; see for instance Kim et al. (2007). However, this "aggregated" estimation approach can become too complex to formulate and to effectively solve in most cases.

When estimating the copula is the primary objective, the unknown marginal distributions of the data enter the problem as unnecessary parameters. The first step is usually to quantify the grades of the marginals using its cumulative distribution functions. In general, the marginal modeling can be done by means of (i) fitting parametric distribution to each marginal, (ii) modeling the marginals nonparametrically using a version of the empirical distribution functions, (iii) using a hybrid of the parametric and nonparametric methods, or (iv) make use of various Bayesian estimators. Indeed, all the mentioned techniques can also be used to estimate parameters of copula functions.

Afterwards, selected marginal distributions cdf's are used to calculate the grades and the copula parameters can be estimated either by Maximum Likelihood Estimation (MLE hereafter) or in some cases calibrated by the Generalized Method of Moments like in Demarta and McNeil (2004). When the copula probability density function is defined, constructing the log likelihood function is straightforward. It is possible to estimate all unknown parameters in one step. Using the MLE method is fairly robust when the number of dimensions are low, but optimization of the likelihood function can become cumbersome when the quantity of estimated parameters increase (this can be seen when dealing with the elliptical copulas where the number of unknown parameters in the correlation matrix can grow quickly). Depending on the copula function representation, MLE can be somehow changed; e.g. when estimating student $t$ copula, correlation matrix and number of degrees of freedom ( $d f$ hereafter) can be estimated simultaneously; or for set $d f$, correlation matrix is estimated. In other words, for every $d f$, MLE is used to find out the correlation matrix. Finally, the resulting values of likelihood function are compared. When estimating special cases of copulas, e.g. student $t$ copulas, the curse of the dimension problem can be solved using the Generalized Method of Moments. The Method of Moments exploits the correspondence between copulas and rank correlations; for detailed discussion about rank correlations see Alexander (2008). For example, it can be shown that Kendall's $\tau$, has a direct relationship with a bivariate copula function $C\left(u_{1}, u_{2}\right)$ as follows:

$$
\tau=4 \int_{0}^{1} \int_{0}^{1} C\left(u_{1}, u_{2}\right) d C\left(u_{1}, u_{2}\right)-1 .
$$

If the copula depends on one parameter then it can be calibrated using a sample estimate of the rank correlation. For instance, the bivariate normal copula depends on one parameter, the correlation coefficient $\rho$, and the above relationship yields:

$$
\rho=\sin \left(\frac{\pi}{2} \tau\right)
$$

This relationship between the sample estimate of Kendall's tau and correlation coefficient holds also for other elliptical copulas, like student t copula. Similar relationships can be also found between rank correlations and non-elliptical copulas in the referenced literature. After specifying the correlation coefficient using the rank correlation, the rest of the 
unknown parameters (e.g. degrees of freedom) are estimated using MLE. The Method of Moments is suitable when dealing with higher dimensionality. All components of the correlation matrix can be thus estimated using the sample Kendall's tau. As in every MLE procedure the best fit from parametric specifications can be determined by Akaike information criterion or Bayesian information criterion.

\section{Conditional Value at Risk optimization}

As was depicted in the above subsection, not only marginal characteristics of financial assets invariants are fat-tailed, their dependency structure is also the source of non-normal behavior. As a consequence new optimization frameworks, which utilize higher moments and extreme risk measures, are used to properly solve portfolio selection problems. Probably the most well-known is the Value at Risk (VaR hereafter) model originally developed by JP Morgan. $\mathrm{VaR}$ is related to the percentiles of loss distributions and measures the predicted maximum loss at a specified probability level over a given horizon and is defined as:

$$
\operatorname{VaR}_{\alpha}\left(R_{P}\right)=-\min \left\{R \mid P\left(R_{P} \leq R\right) \geq \alpha\right\},
$$

where $P$ denotes the probability function, $R_{P}$ represent's the portfolio's expected return over a given horizon and $\alpha$ is a chosen confidence level. In portfolio selection, rational investors choose a portfolio that minimizes $\mathrm{VaR}$ for targeted expected returns. The optimization problem can also be reversed. The investor maximizes expected returns for targeted values of VaR. A more detail description of the $\mathrm{VaR}$ framework can be found in Jorion (2007).

As was stated in the introductory section, VaR has several undesirable properties, which has led to many extensions of the VaR framework. One of the most popular is Conditional Value at Risk (CVaR). With no loss in generality, the $\mathrm{CVaR}$ can be defined as:

$$
\operatorname{CVaR}_{\alpha}\left(R_{P}\right)=-E\left(R_{P} \mid R_{P} \leq-\operatorname{VaR}_{\alpha}\left(R_{P}\right)\right) \text {. }
$$

$\mathrm{CVaR}$ is by definition always at least as large as $\mathrm{VaR}$ and it is a coherent risk measure by means of Artzner et al. (1999). It can be shown that CVaR is a concave function and, therefore has a unique minimum. The resulting optimization problem takes the form

$$
\max _{w} \mu^{\prime} w
$$

subject to

$$
\begin{gathered}
\operatorname{CVaR}_{\alpha}(w) \leq C_{0}, \\
w \in C_{w},
\end{gathered}
$$

where $\mu$ represents the vector of expected returns, $w$ are optimal portfolio weights, while $C_{0}$ and $C_{w}$ are sets of constrains. Using the expression in (19) to estimate CVaR involves knowing the VaR function representation. This can become ineffective in largesize problems. Rockafellar and Uryasev (2000) proposed a simpler approach where $\mathrm{CVaR}$ is reformulated as a minimizer of the auxiliary function $F_{\alpha}$. Their approach is particularly suitable when the probability density function is expressed by Monte Carlo simulations and hence the portfolio selection problem can be solved as linear programming problem. For a description of the resulting optimization problem refer to Rockafellar and Uryasev (2000) or Meucci (2006).

\section{Copulas in portfolio management}

The crucial role of copulas in modeling the behavior of portfolio, which invests in derivative instruments, is clear. The pay off of derivatives is usually non-linear and so too are dependencies between derivatives and underlying assets. Therefore the use of copulas in that field of study is natural. When the portfolio invests only in stocks and bonds, and is possibly exposed to non-negative weight constraints, the need for copula formulation of dependence structure is not obvious. The examples from the second part intuitively support the idea of copula modeling in regular, long-only portfolio management. Most of the presented gradegrade charts underline the empirical observation from financial markets which is sometimes called lack of diversification. In the exceptionally negative market shocks nearly all assets perform in the same fashion. The financial markets stress from the second half of 2008 is the latest case.

In this section we compare the differences in optimal portfolios that are estimated using two different copula specifications - normal copula and student $t$ copula. The optimal portfolio problem was solved by means of mean - CVaR optimization briefly introduced in a previous section. The rational behind using $\mathrm{CVaR}$ as a risk measure is its implicit focus on optimal portfolios' tail characteristics. Consequently, there is supposed to be a relationship between different copula assumptions and different CVaR confidence levels. If the empirical dependency structure exhibits fat tails - student $t$ copula better describes these relationships - optimal portfolios should be more distinct for higher confidence levels. In other words, the deeper we look into the distributional tails, the more evident the difference between created portfolios should be, when normal and student $t$ copula assumptions take place. 
We considered an investment entity that was assumed to be a long-only mutual or a pension fund which invests in 3 bond market indexes and 6 stock indexes. The bond investments were represented by iBoxx ${ }^{3}$ indexes and cover US Treasuries, the Euro Government Bond Market and the Euro A Corporate Bond Market. Considered stock investments were: S\&P 500, DJ Stoxx 600, CECE Traded Eur Index, Nikkei 225, NASDAQ 100 and Hang Seng China Enterprises. All calculations were done using weekly data from $15^{\text {th }}$ January 1999 to $24^{\text {th }}$ July 2009 , which add up to 550 weekly observations of return data. The detailed statistical description of data is presented in appendix C. In the case of bond investments we adopted a more elementary approach than is usually presented in literature. Bond market invariants were not used directly but returns of total return indexes were used. The proposed simplification is legitimated by two reasons: firstly, there can be found investment vehicles that offer investors the chance of exposure to fixed-income indexes directly via exchange traded funds or mutual funds. Their total returns could be assumed as market invariants. Secondly and more importantly, portfolio construction problem perspective enables us to demand lower precision as many more errors arise from stochastic estimates. The global asset allocation is focused on estimating optimal risk exposures for given expected returns whereas investment vehicle selections are subject to tactical asset allocation. As is pointed out by Jorion (2007), the risk management and derivative valuation approaches have much methodology in common. However, valuation methods require more precision as accurate asset prices are needed for trading purposes. We suggest that the portfolio construction problem, specifically global asset allocation, enables us to use less precision as well and focus on overall exposures.

Firstly, we isolated marginal distributions using empirical cumulative distribution functions $F^{E}(x)$. They were further smoothed by means of the Gaussian kernel function to obtain more spaced observations as in Meucci (2006). Thus the skewness and/or the kurtosis of marginal distributions were preserved. The parameters to be estimated remained correlation matrix in the case of normal copula and correlation matrix and degrees of freedom in the case of student $t$ copula. The estimation of normal copula correlation matrix is straightforward, as is its MLE sample estimator. Recall that the copula operates on grades or percentiles of underlying distributions. The estimation of normal copula and simulation from its distribution can be visualized as in Figure 5.

\begin{tabular}{|ll|}
\hline$X$ & $Y$ \\
$\downarrow F^{E}(x)$ & $\uparrow F^{E-1}(x)$ \\
$U$ & $U$ \\
$\downarrow \Phi^{-1}$ & $\uparrow \Phi$ \\
$X^{\text {copula }} \stackrel{\text { estimation }}{\longrightarrow} \hat{\Sigma} \stackrel{\text { simulation }}{\longrightarrow}$ & $Y^{\text {copula }}$ \\
\hline
\end{tabular}

Figure 5 Copula modeling framework

The $X(550 \times 9)$ matrix consists of weekly observations of nine considered investments. $X$ was transformed to equally distributed grades' matrix $U$ using empirical cdf functions. Standard normal inverse function was further used to create $X^{\text {copula }}$ matrix from which correlation matrix was estimated. The parameters of student $t$ copula were estimated following the same steps. Obviously we used student $t$ inverse function. Several values of $d f$ were chosen and for every assumed number, we estimated correlation matrix. Having set number of $d f$ and correlation matrix, the log likelihood function was calculated. However, the local maximum was not found. Firstly, the values of the log likelihood increased quickly when the number of $d f$ was small, but after reaching the level of 9 its values became not elastic to additional changes in number of $d f$. Using this number of $d f$ represented approximately $95 \%$ of the total improvement in the log likelihood function. For further analysis we considered 9 as the estimated number of $d f$.

After specifying the parameters of normal and student t copula, we generated 10000 Monte Carlo simulations from corresponding copula distribution using algorithms proposed in Alexander (2008) and visualized in the right hand side of Figure 5. $Y^{\text {copula }}$ $(10000 \mathrm{x}$ 9) matrix represents Monte Carlo simulations which were firstly transformed to equally distributed grades' matrix $U$ using normal or student $t$ cdf functions. The inverse empirical cumulative density functions were finally used to calculate weekly returns, panel $Y$. To get rid of unstable expected returns estimate, simulated sample averages of $Y$ were adjusted to implied returns that were induced by empirical covariance matrix; assumed market portfolio weights $w^{\text {market }}$ and assumed investors risk aversion coefficient $\lambda$. The implied returns vector $\mu^{I M P L}$ is defined as:

$$
\mu^{I M P L}=\lambda \Sigma^{E} w^{\text {market }} .
$$

The concepts of reverse optimization and implied returns were developed by Sharpe (1974) and are widely used in Black-Litterman models. Standard pension fund benchmark portfolio was assumed as $w^{\text {market }}$. Risk aversion coefficient was set to 4 .

\footnotetext{
${ }^{3}$ www.indexco.com or www.markit.com.
} 
Obviously, separate simulation panels were created for both copula assumptions.

Simulated panels $Y$ were used to estimate optimal portfolios by solving (20). We estimated values of $\mathrm{CVaR}$ as a sample average loss that exceeded corresponding VaR. We defined $\mathrm{VaR}$ as empirical quantile of simulated portfolio returns. Firstly, a portfolio with minimum attainable $\mathrm{CVaR}$ was estimated. Afterwards, we reversed the optimization setting and estimated optimal portfolios by maximizing expected returns for targeted CVaR. The CVaR targets equalled to minimum $\mathrm{CVaR}$ subsequently increased by $0.25 \%$ up to the optimal portfolio equity allocation reached unity (this examination criterion will be introduced shortly). We estimated optimal portfolios with three different CVaR confidence levels: $1 \%, 3 \%$ and $5 \%$.

As an examination criterion we adopted the difference in the optimal portfolios' total weights of equity investments for the same CVaR target. Since the optimal portfolios were estimated from different simulated panels $Y$, it was not possible to use formal statistical tests to evaluate their correspondence.

Following empirical observations we can conclude that equity investments usually suffer from heavy tails and strong lower tail dependence. So student $t$ copula is supposed to capture these effects and should lead to more conservative optimal portfolios. Figure 6 shows difference curves for chosen confidence levels. They represent equity allocation differences between normal and student $t$ copula.

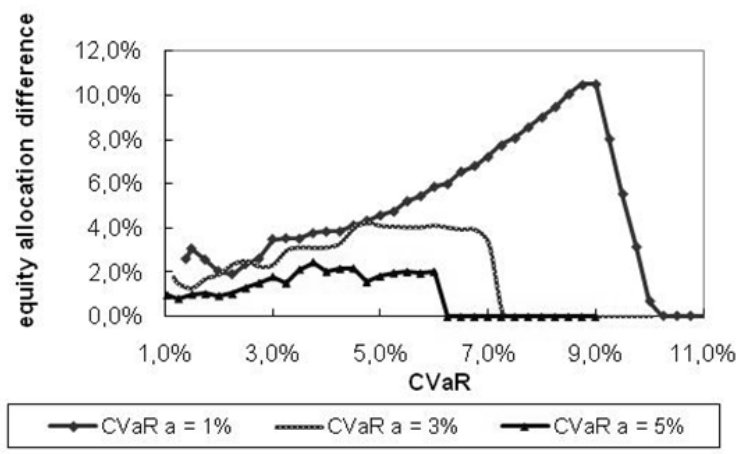

\section{Figure 6 Dierenceurves}

As Figure 6 shows, optimal portfolios estimated under normal copula assumption often leads to higher allocation into equity investments. We point out that allocation differences are solely the result of dependency structure assumptions as the marginal characteristics are treated equally. The minimum attainable CVaR targets were $1.04 \%$ in the case of $5 \%$ confidence level, $1.15 \%-1.16 \%$ in the case of $3 \%$ confidence level and $1.36 \%-1.40 \%$ in the last case. As was stated above, CVaR targets were further increased by $0.25 \%$ and portfolios were optimized according to these values. Full investments into equities under student $\mathrm{t}$ copula assumption were reached with $\mathrm{CVaR}$ target set to $6.25 \%(\alpha=5 \%)$, $7.5 \%(\alpha=3 \%)$ and $10.25 \%(\alpha=1 \%)$. Normal copula leads to full equity allocations significantly sooner. The 5\% confidence level difference curve is the flattest. The equity allocations do not differ by more than $2.5 \%$. However, the more risky profile of optimal portfolios estimated under normal copula assumption became more apparent when increasing $\mathrm{CVaR}$ confidence levels. As expected, growing CVaR confidence level intensifies conceptual differences between copula assumptions.

This result has strong consequences as a rather high confidence level of $1 \%$ is required for internal risk models by the Basel Supervisory Committee. So it is highly recommended to investigate the possibility of using different dependency structures when assessing capital adequacy requirements. The $1 \%$ confidence level difference curve gives another insight as well. The largest differences are reached when the most aggressive CVaR targets are selected. The same explanation as in the case of confidence level changes is valid. Higher $\mathrm{CVaR}$ targets focus on deeper distributional tails where differences between normal and student $t$ copula assumptions are more striking. On the other hand, high CVaR targets are usually only used by risk seeking mutual funds.

Different copula assumptions resulted in different individual positions as well. Table 2 shows estimated portfolios' weights for several optimization targets and confidence levels. These differences can easily lead to the misunderstanding of proper risk exposures and ineffective hedging scheme. Compositions of other estimated optimal portfolios are available upon request.

\section{Conclusion}

The copula functions can help to realistically describe the true dependencies among random numbers. Moreover, they propose a practical solution to the lack of diversification problem. The term copula modeling framework was used throughout the article and its basic concepts were described. The most important feature is the ability to model non-linear dependencies among market invariants. Additionally, it enables us to separately model marginal characteristics and dependency structures. Different statistical and econometrical tools can be used for both parts. There exist many different classes of copulas. For modeling fat-tailed dependencies, the elliptical class of copulas is usually sufficient. When the asymmetric dependencies are present, the 
Archimedean copulas are a better choice. The potential problem is the estimation of exact parametric specification of copula function when the number of dimensions is large.

The portfolio selection problem defined in terms of Conditional VaR optimization enables us to fully utilize copula modeling framework while the meeting requirements of coherent risk measure. Despite its linear representation, the efficiently solution to the CVaR problem is rather complex.

The presented examples showed better properties of student $t$ copula than normal in the field of portfolio management. Optimal portfolios estimated under normal copula assumption often lead to higher allocation into equity investments that usually suffer from heavy tails and strong lower tail dependence. The extent of equity allocation overweight proved to be a function of CVaR targets and CVaR confidence levels. Both higher confidence levels and targets intensified the differences between assumed copula specifications. Our results recommend focusing on advanced dependency structure models in various fields of financial modeling.

\section{References}

ALEXANDER, C. (2008). Market Risk Analysis: Practical Financial Econometrics. Hoboken NJ: Wiley.
ARTZNER, P., DELBAEN, F., EBER, J.M., HEATH, D. (1999). Coherent Measures of Risk. The Journal of Mathematical Finance 9: 203-228.

http://dx.doi.org/10.1111/1467-9965.00068

BAI, M., SUN, L. (2007). Application of Copula and Copula-CVaR in the Multivariate Portfolio Optimization. In: Chen, B., Paterson, M., Zhang, G. (eds.): Combinatorics, Algorithms, Probabilistic and Experimental Methodologies, 231-242. Berlin/ Heidelberg: Springer. http://dx.doi.org/10.1007/978-3540-74450-4

BOUYÉ, E., DURRLEMAN, V., NIKEGHBALI, A., RIBOULET, G., RONCALLI, T. (2000). Copulas for finance: A Reading Guide and Some Applications. Groupe de Recherche Operationnelle, Credit Lyonnais. Working Paper.

OLES, S., HEFFERNAN, J., TAWN, J. (1999). Dependence Measures for Extreme Value Analyses. Extremes 2: 339-365.

http://dx.doi.org/10.1023/A:1009963131610

DEMARTA, S., McNEIL, A.J. (2004). The t Copula and Related Copulas. International Statistical Review 73: $\quad 111-129 . \quad$ http://dx.doi.org/10.1111/j.17515823.2005.tb00254.x

EMBRECHTS, P., McNEIL, A.J., STRAUMANN, D. (2002). Correlation and dependence in risk management: Properties and pitfalls. In.: Dempster, M. (eds.): Risk Management: Value at Risk and Beyond, 176-223. Cambridge: Cambridge University Press. http://dx.doi.org/10.1017/CBO9780511615337

Table 2 Composition of selected optimal portfolios

\begin{tabular}{|c|c|c|c|c|c|c|c|c|c|c|c|c|}
\hline Copula & Student & Normal & Student & Normal & Student & Normal & Student & Normal & Student & Normal & Student & Normal \\
\hline$\alpha$ & \multicolumn{2}{|c|}{$1 \%$} & \multicolumn{2}{|c|}{$3 \%$} & \multicolumn{2}{|c|}{$5 \%$} & \multicolumn{2}{|c|}{$1 \%$} & \multicolumn{2}{|c|}{$3 \%$} & \multicolumn{2}{|c|}{$5 \%$} \\
\hline CVaR & \multicolumn{6}{|c|}{$-1.5 \%$} & \multicolumn{6}{|c|}{$-4.0 \%$} \\
\hline $\begin{array}{l}\text { QW1A } \\
\text { Index }\end{array}$ & $74.2 \%$ & $66.0 \%$ & $49.3 \%$ & $39.0 \%$ & $47.5 \%$ & $27.1 \%$ & $0.0 \%$ & $0.0 \%$ & $0.0 \%$ & $0.0 \%$ & $0.0 \%$ & $0.0 \%$ \\
\hline $\begin{array}{l}\text { ITRROV } \\
\text { Index } \\
\end{array}$ & $12.7 \%$ & $17.7 \%$ & $27.0 \%$ & $32.2 \%$ & $16.1 \%$ & $33.9 \%$ & $43.4 \%$ & $24.7 \%$ & $21.7 \%$ & $29.8 \%$ & $21.1 \%$ & $28.7 \%$ \\
\hline QX5N Index & $0.0 \%$ & $0.1 \%$ & $2.7 \%$ & $6.5 \%$ & $11.6 \%$ & $13.2 \%$ & $14.5 \%$ & $29.4 \%$ & $21.8 \%$ & $10.5 \%$ & $12.5 \%$ & $2.9 \%$ \\
\hline S\&P 500 & $4.30 \%$ & $4.57 \%$ & $5.42 \%$ & $7.14 \%$ & $7.48 \%$ & $9.71 \%$ & $10.49 \%$ & $14.11 \%$ & $16.54 \%$ & $21.75 \%$ & $22.85 \%$ & $27.43 \%$ \\
\hline $\begin{array}{l}\text { DJ STOXX } \\
600\end{array}$ & $6.93 \%$ & $4.78 \%$ & $11.56 \%$ & $8.97 \%$ & $11.18 \%$ & $8.83 \%$ & $15.84 \%$ & $14.02 \%$ & $18.90 \%$ & $15.96 \%$ & $18.93 \%$ & $16.82 \%$ \\
\hline CECE-EUR & $0.00 \%$ & $2.08 \%$ & $0.54 \%$ & $1.26 \%$ & $1.29 \%$ & $2.11 \%$ & $3.40 \%$ & $5.63 \%$ & $5.63 \%$ & $8.37 \%$ & $8.13 \%$ & $9.07 \%$ \\
\hline NIKKEI 225 & $1.35 \%$ & $1.89 \%$ & $2.27 \%$ & $3.00 \%$ & $3.14 \%$ & $3.37 \%$ & $2.91 \%$ & $5.69 \%$ & $6.70 \%$ & $6.58 \%$ & $9.11 \%$ & $7.45 \%$ \\
\hline $\begin{array}{l}\text { NASDAQ } \\
100 \\
\end{array}$ & $0.07 \%$ & $1.11 \%$ & $0.00 \%$ & $0.00 \%$ & $0.14 \%$ & $0.00 \%$ & $2.20 \%$ & $0.02 \%$ & $3.01 \%$ & $0.00 \%$ & $0.95 \%$ & $0.00 \%$ \\
\hline \begin{tabular}{|l|} 
HSCEI \\
Index \\
\end{tabular} & $0.40 \%$ & $1.67 \%$ & $1.15 \%$ & $1.88 \%$ & $1.51 \%$ & $1.72 \%$ & $7.25 \%$ & $6.44 \%$ & $5.69 \%$ & $6.98 \%$ & $6.41 \%$ & $7.61 \%$ \\
\hline $\begin{array}{l}\text { equity } \\
\text { allocation }\end{array}$ & $13.1 \%$ & $16.1 \%$ & $20.9 \%$ & $22.3 \%$ & $24.7 \%$ & $25.7 \%$ & $42.1 \%$ & $45.9 \%$ & $56.5 \%$ & $59.6 \%$ & $66.4 \%$ & $68.4 \%$ \\
\hline
\end{tabular}


EMBRECHTS, P. (2008). Copulas: A personal view. Journal of Risk and Insurance 76: 639-650. http://dx.doi.org/10.1111/j.1539-6975.2009.01310.x

GENEST, C., FAVRE, A.C. (2007). Everything you always wanted to know about copula modeling but were afraid to ask. Journal of Hydrologic Engineering 12: 347-368. http://dx.doi.org/10.1061/(ASCE)10840699(2007)12:4(347)

FABOZZI , F.J., FOCARDI, S.M., KOLM, P.N. (2006). Financial Modeling of the Equity Market: From CAPM to Cointegration. Hoboken NJ: Wiley.

HE, X., GONG, P. (2009). Measuring the coupled risks: A copula-based CVaR model. Journal of computational and applied mathematics 223: 10661080. http://dx.doi.org/10.1016/j.cam.2008.03.046

CHERUBINI, U., LUCIANO, E., VECCHIATO W. (2004). Copula methods in finance. Hoboken NJ: Wiley.

CHORÓS, B., HÄRDLE, W., OKHRIN, O. (2009). CDO Pricing with Copulae. SFB 649 Discussion Paper, 2009 - 013, Humbolt - Universität zu Berlin.

JORION, P. (2007). Value-at-Risk: The New Benchmark for Managing Financial Risk, $3^{\text {rd }}$ edition. New York: McGraw-Hill.

KIM, G., SILVAPULLE, M.J., SILVAPULLE, P. (2007). Comparison of semiparametric and parametric methods for estimating copulas. Computational Statistics \& Data analysis 51: 2836-2850. http://dx.doi.org/10.1016/j.csda.2006.10.009
MEUCCI, A. (2005). Risk and Asset Allocation. New York: Springer. http://dx.doi.org/10.1007/978-3-54027904-4

MEUCCI, A. (2006). Beyond Black-Litterman in Practice. Risk Magazine 19: 114-119.

NELSEN, R.B. (2006). An introduction to Copula, $2^{\text {nd }}$ edition. New York: Springer.

PALARO, H.P., HOTTA, L.K. (2006). Using Conditional Copula to Estimate Value at Risk. Journal of Data Science 4: 93-115.

RAU-BREDOW, H. (2004). Value-at-Risk, Expected Shortfall and Marginal Risk Contribution. In: Szego, G. (eds.): Risk Measures for the $21^{\text {st }}$ Centrury, 61-67. Hoboken NJ: Wiley.

ROCKAFELLAR, R., URYASEV, S. (2000). Optimization of Conditional Value-At-Risk. The Journal of Risk 2: 21-41.

SHARPE, W.F. (1974). Imputing expected returns from portfolio composition. Journal of Financial and Quantitative Analysis June 1974: 463-472. http://dx.doi.org/10.2307/2329873

STULZ, R. (2008). Risk Management Failures: What are They and When Dot They Happen?. Fisher College of Business Working Paper Series. October 2008.

\section{Appendix A}

Table A1 Relative frequencies of different dependency structure assumptions using daily data

\begin{tabular}{|l|c|c|c|c|c|c|c|}
\hline & & \multicolumn{3}{|c|}{ Empirical tail frequency } & \multicolumn{3}{|c|}{ Normal copula tail frequency } \\
\hline Invariants pair & $\rho$ & 5 th -5 th & 10 th -10 th & 20 th -20 th & 5 th -5 th & 10 th -10 th & 20 th -20 th \\
\hline S\&P 500 - DJ STOXX 600 & 0.562 & $2.47 \%$ & $5.01 \%$ & $9.94 \%$ & $1.40 \%$ & $3.44 \%$ & $9.16 \%$ \\
\hline S\&P 500 - NASDAQ 100 & 0.956 & $4.08 \%$ & $8.40 \%$ & $16.95 \%$ & $3.71 \%$ & $7.88 \%$ & $17.02 \%$ \\
\hline S\&P 500 - NIKKEI 225 & 0.524 & $0.85 \%$ & $1.85 \%$ & $5.32 \%$ & $1.24 \%$ & $3.27 \%$ & $8.80 \%$ \\
\hline DJ STOXX 600 - NIKKEI 225 & 0.418 & $1.62 \%$ & $3.47 \%$ & $8.09 \%$ & $1.05 \%$ & $2.72 \%$ & $7.81 \%$ \\
\hline US 2YR TR - US 10YR TR & 0.798 & $2.77 \%$ & $6.39 \%$ & $14.02 \%$ & $2.52 \%$ & $5.31 \%$ & $12.81 \%$ \\
\hline GE 10YR TR - US 10YR TR & 0.515 & $2.00 \%$ & $4.16 \%$ & $10.94 \%$ & $1.24 \%$ & $3.22 \%$ & $8.74 \%$ \\
\hline CECEEUR - DJ STOXX 600 & 0.723 & $2.70 \%$ & $6.24 \%$ & $12.87 \%$ & $2.06 \%$ & $4.72 \%$ & $11.46 \%$ \\
\hline S\&P 500 - US 10YR TR & 0.379 & $2.23 \%$ & $4.47 \%$ & $8.94 \%$ & $0.89 \%$ & $2.52 \%$ & $7.36 \%$ \\
\hline
\end{tabular}




\section{Appendix B}

In this appendix different grade-grade charts of different financial data series are shown. Weekly as well as daily data are used. The relative frequencies of weekly data series is included in section 2 and that of daily data in Appendix A.

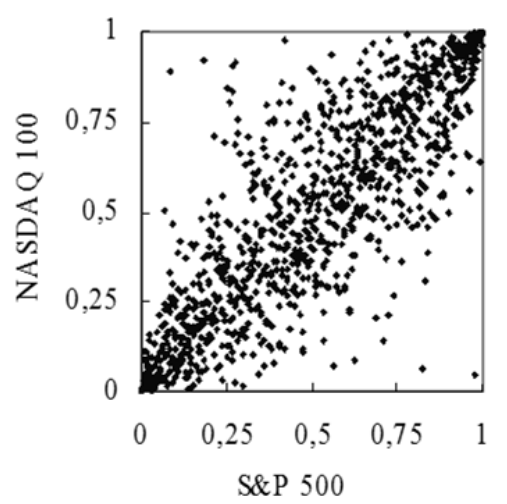

Figure B1 Grade-grade chart of S\&P 500 and NASDAQ $100-$ weekly data

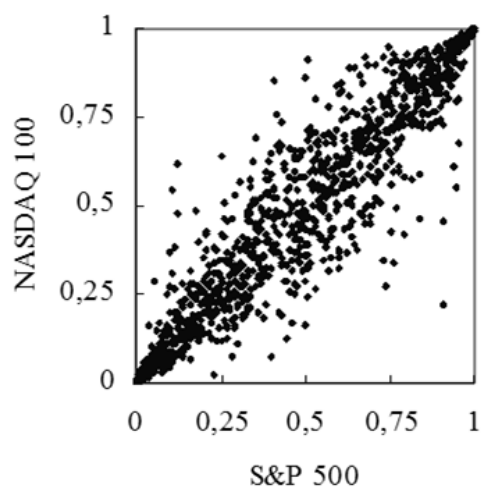

Figure B4 Grade-grade chart of S\&P 500 and NASDAQ 100 - daily data

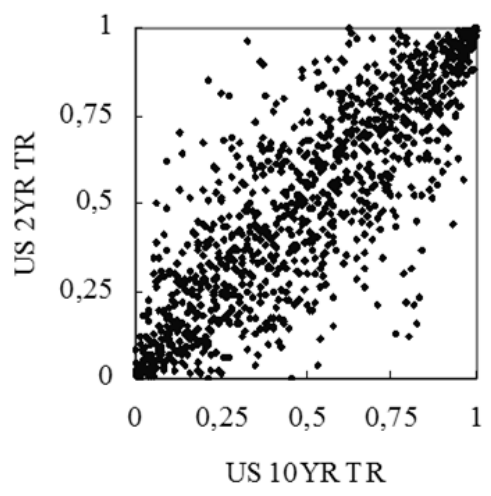

Figure B2 Grade-grade chart of US 2YR and US 10 YR Total Return treasury bond yields - weekly data

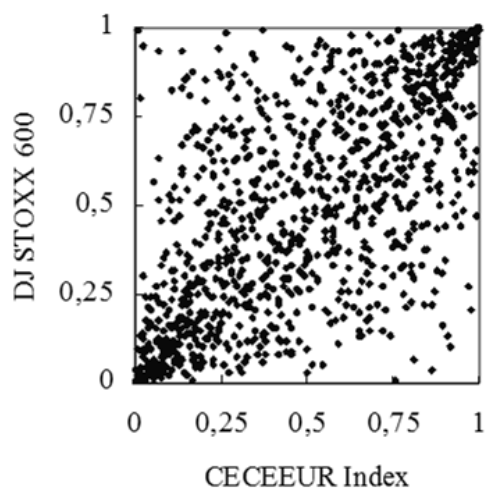

Figure B5 Grade-grade chart of DJ Stoxx 600 and CECEEUR Index-daily data

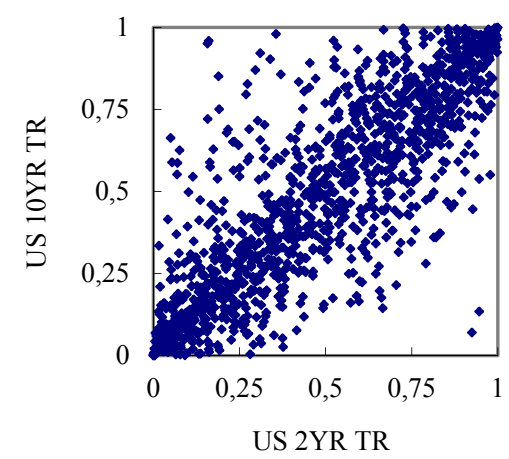

Figure B7 Grade-grade chart of US 2YR and US 10 YR Total Return treasury bond' yields - daily data

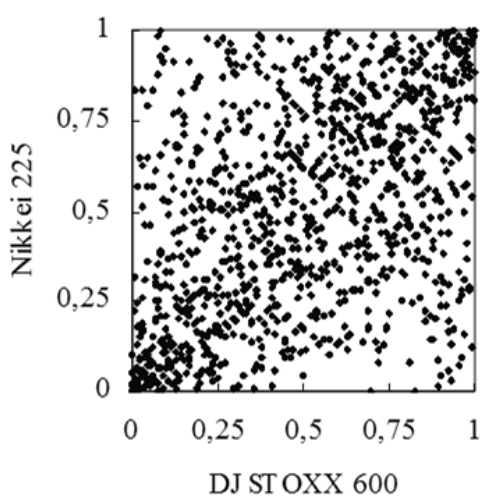

Figure B3 Grade-grade chart of Nikkei 225 and DJ Stoxx 600 - weekly data

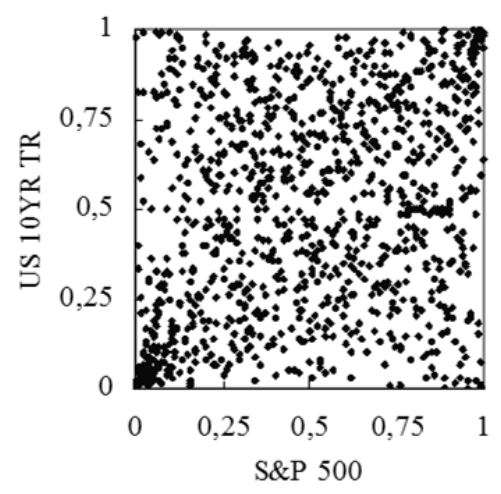

Figure B6 Grade-grade chart of S\&P 500 and US 10 YR Total Return treasury bond' yields - daily data 


\section{Appendix C}

In this appendix description of different date sets is presented. Table $\mathrm{C} 1$ shows statistics of 6 financial time series which represent 4 equity market indices and 2 fixed-income market invariants. The sampling frequency is weekly for all series and includes time window from $6^{\text {th }}$ January 1989 to $17^{\text {th }}$ July 2009 , totaling to 1072 observations.

Table C1 Description of data used to calculate quantities from Table 1

\begin{tabular}{|l|c|c|c|c|c|c|}
\hline & S\&P 500 & DJ STOXX 600 & NASDAQ 100 & NIKKEI 225 & US 2YR TR & US 10YR TR \\
\hline$\mu$ & $0.11 \%$ & $0.08 \%$ & $0.15 \%$ & $-0.11 \%$ & -0.76 bps. & $-0.51 \mathrm{bps}$. \\
\hline$\mu$ p.a. & $5.92 \%$ & $4.30 \%$ & $7.76 \%$ & $-5.66 \%$ & - & - \\
\hline s & $2.36 \%$ & $2.48 \%$ & $3.27 \%$ & $3.10 \%$ & $14.22 \mathrm{bps}$. & $13.14 \mathrm{bps}$. \\
\hline s p.a. & $17.00 \%$ & $17.86 \%$ & $23.59 \%$ & $22.33 \%$ & 102.58 bps. & $94.77 \mathrm{bps}$. \\
\hline skew & -0.82 & -1.01 & -1.00 & -0.78 & 0.03 & 0.33 \\
\hline kurt & 7.59 & 10.95 & 8.59 & 6.86 & 1.57 & 0.73 \\
\hline N & 1072 & 1072 & 1072 & 1072 & 1072 & 1072 \\
\hline min & $-20.10 \%$ & $-24.30 \%$ & $-29.20 \%$ & $-27.90 \%$ & $-63.90 \mathrm{bps}$. & $-53.75 \mathrm{bps}$. \\
\hline max & $11.40 \%$ & $12.40 \%$ & $17.40 \%$ & $11.40 \%$ & $65.82 \mathrm{bps}$. & $54.00 \mathrm{bps}$. \\
\hline VaR 5\% & $-3.54 \%$ & $-3.67 \%$ & $-5.07 \%$ & $-4.98 \%$ & $-23.45 \mathrm{bps}$. & $-20.25 \mathrm{bps.}$ \\
\hline CVaR 5\% & $-5.62 \%$ & $-5.90 \%$ & $-7.93 \%$ & $-7.18 \%$ & $-31.87 \mathrm{bps}$. & $-25.56 \mathrm{bps.}$ \\
\hline
\end{tabular}

Table C2 depicts statistics of financial data series used in section 4. As in previous table, data represent both fixed-income and equity market series sampled weekly. Instead of directly using fixed-income invariants, total return indices were used as proxy for bond markets. The time window represent period from $15^{\text {th }}$ January 1999 to $24^{\text {th }}$ July 2009.

Table C2 Description of data used in examples from section 4

\begin{tabular}{|l|c|c|c|c|c|c|c|c|c|}
\hline & $\begin{array}{c}\text { QW1A } \\
\text { Index }\end{array}$ & $\begin{array}{c}\text { ITRROV } \\
\text { Index }\end{array}$ & $\begin{array}{c}\text { QX5N } \\
\text { Index }\end{array}$ & S\&P 500 & $\begin{array}{c}\text { DJ } \\
\text { STOXX } \\
600\end{array}$ & CECEEUR & $\begin{array}{c}\text { NIKKEI } \\
225\end{array}$ & $\begin{array}{c}\text { NASDAQ } \\
100\end{array}$ & $\begin{array}{c}\text { HSCEI } \\
\text { Index }\end{array}$ \\
\hline$\mu$ & $0.09 \%$ & $0.10 \%$ & $0.07 \%$ & $-0.05 \%$ & $-0.05 \%$ & $0.11 \%$ & $-0.05 \%$ & $-0.03 \%$ & $0.35 \%$ \\
\hline$\mu$ p.a. & $4.47 \%$ & $5.36 \%$ & $3.38 \%$ & $-2.50 \%$ & $-2.82 \%$ & $5.56 \%$ & $-2.81 \%$ & $-1.66 \%$ & $18.25 \%$ \\
\hline$\sigma$ & $0.51 \%$ & $0.66 \%$ & $0.55 \%$ & $2.79 \%$ & $2.90 \%$ & $3.99 \%$ & $3.22 \%$ & $4.03 \%$ & $5.15 \%$ \\
\hline$\sigma$ p.a. & $3.71 \%$ & $4.76 \%$ & $3.98 \%$ & $20.12 \%$ & $20.89 \%$ & $28.77 \%$ & $23.22 \%$ & $29.03 \%$ & $37.13 \%$ \\
\hline skew & -0.29 & -0.39 & -1.59 & -0.82 & -1.13 & -0.63 & -1.39 & -0.89 & -0.24 \\
\hline kurt & 0.68 & 1.07 & 9.12 & 6.72 & 10.31 & 6.40 & 10.24 & 6.35 & 1.56 \\
\hline $\mathrm{N}$ & 550 & 550 & 550 & 550 & 550 & 550 & 550 & 550 & 550 \\
\hline min & $-1.80 \%$ & $-3.00 \%$ & $-4.40 \%$ & $-20.10 \%$ & $-24.30 \%$ & $-26.60 \%$ & $-27.90 \%$ & $-29.20 \%$ & $-23.40 \%$ \\
\hline max & $1.70 \%$ & $2.40 \%$ & $1.40 \%$ & $11.40 \%$ & $12.40 \%$ & $22.90 \%$ & $11.40 \%$ & $17.40 \%$ & $18.00 \%$ \\
\hline $\begin{array}{l}\text { VaR } \\
5 \%\end{array}$ & $-0.79 \%$ & $-1.01 \%$ & $-0.83 \%$ & $-4.44 \%$ & $-4.79 \%$ & $-6.13 \%$ & $-5.24 \%$ & $-6.28 \%$ & $-8.47 \%$ \\
\hline $\begin{array}{l}\text { CVaR } \\
5 \%\end{array}$ & $-1.09 \%$ & $-1.46 \%$ & $-1.32 \%$ & $-6.85 \%$ & $-7.12 \%$ & $-9.70 \%$ & $-7.58 \%$ & $-9.68 \%$ & $-11.47 \%$ \\
\hline
\end{tabular}

* QW1A Index - iBoxx € Sovereigns Eurozone, ITRROV Index - iBoxx \$ Treasuries Total Return Index, QX5N Index iBoxx € Corporates A, HSCEI Index - HANG SENG CHINA ENT INDX 